\title{
Matrixmetalloproteinase-9 Genetic Variation with Cataract, Glaucoma and Retinoblastoma in a Senegalese Population
}

\author{
Matar Ciss \\ Genetic for Gestion of Population team (GENGESPOP), Department of Animal Biology, Faculty of sciences and Technics, Cheikh Anta Diop \\ University, Dakar, Sénégal \\ Ndéye Ndoumbé Guéye \\ Ophthalmology Department, Principal Hospital, Dakar, Senegal \\ Fatimata Mbaye* \\ Genetic for Gestion of Population team (GENGESPOP), Department of Animal Biology, Faculty of sciences and Technics, Cheikh Anta Diop \\ University, Dakar, Sénégal \\ Paul Aïda Ndoye \\ Ophthalmology Department, Aristide Le Dantec Hospital, Dakar, Senegal \\ Chérif Dial \\ Anapathologic Department, Grand Yoff General Hospital, Dakar, Senegal \\ Joseph Ndiaye \\ Ophthalmology Department, Aristide Le Dantec Hospital, Dakar, Senegal
}

\section{Mbacké Sembéne}

Genetic for Gestion of Population team (GENGESPOP), Department of Animal Biology, Faculty of sciences and Technics, Cheikh Anta Diop University, Dakar, Sénégal

\begin{abstract}
The Matrix metalloproteinase-9 is involved in several pathologies. Its strong presence in ocular pathologies explains our interest for its genetic variation in cataract, glaucoma and retinoblastoma in Senegal. MMP9 is highly polymorphic with cataract and glaucoma. 77 mutations were noted with 21 haplotypes for the entire population. The haplotype diversity $\mathrm{Hd}$ is 0.831 and the nucleotide diversity $\mathrm{Pi}$ is $0.016 ; \mathrm{k}=17.395$. The polymorphism of the Matrix metalloproteinase-9 gene is associated with all three diseases and SNP 3918249 is found in both cataract and glaucoma.

Keywords: Matrix metalloproteinase-9; Cataract; Glaucoma; Polymorphism.

(9) (i) CC BY: Creative Commons Attribution License 4.0

\section{Introduction}

MMPs are proteins that have a variable combination of different modules on the same peptide chain [1]. MMP9 is an enzyme also known as $92 \mathrm{KDa}$ type IV collagenase, $92 \mathrm{KDa}$ gelatinase or gelatinase $\mathrm{B}$ (GElB).

As for the MMP, it is expressed by the trophoblast cells which invade the maternal tissues during the embryonic implantation [2] and by the osteoclasts during the endochondral ossification [3].

In adulthood the MMP is mainly produced by the inflammatory cells (eosinophils, neutrophils, monocytes, macrophages, lymphocytes B and T), some epithelial cells (bronchial epithelium) and some tumoral cells. An increase of its expression was noticed during certain diseases; tumoral invasion (rheumatoid arthritis, asthma, Alzheimer disease); ossification and in remodeling the extracellular matrix (ECM) during homeostasis and healing. It is present in intraocular areas such as the aqueous humor and is more highly expressed in glaucomatous eyes.

Ocular diseases have a fairly strong penetrance in the Senegalese population, which motivates our interest and encourages us to make a genetic study which can detect their molecular expression in the said population. One gene was isolated for this study, MMP9. Cataract and Glaucoma are diseases that can be sporadic or family. The search for neomutations specific to the Senegalese population studied is one of the key objectives of our study. In molecular analysis we will opt as an approach, the study of the polymorphism and the gene diversity.
\end{abstract}

\section{Material and Method}

\subsection{Patients}

The study involves forty eights (48) blood samples from patients with cataract (35), glaucoma (6) and controls (07) collected at the hospital Aristide le Dantec (HALD), at the hospital principal de Dakar (HPD), Grand Yoff 
General Hospital (HOGGY) and Ouakam Military Hospital (HMO). Samples were taken from these patients after mutual consent of the latter and these hospital structure ethics committees. Samples were stored in $95 \%$ alcohol.

\subsection{Methods}

The total DNA tissue was extracted using the Standard Qiagen (Qiagen Dneasy Blood Kit) method. The MMP9 gene has been amplified. The amplifications are carried out in a reaction volume of $50 \mu \mathrm{l}$. PCR products are controlled by electrophoretic migration on agarose gel of $1.5 \%$ from $5 \mu$ l of them with $3 \mu$ of bromophenol blue. The size of the amplified gene is estimated using a SmartLadder 200pb size marker. After UV visualization, the PCR products for which the primers have hooked up are frozen in $1.5 \mathrm{ml}$ Eppendorf tubes for sequencing reasons.

As part of the genetic study of the three diseases we will start with a polymorphic approach of the $M M P 9$ gene through the application Mutation Surveyor, Mega 6 and Dnasp which are used to determine the number of mutations present in the studied gene portions.

Polymorphism will edify us on the molecular identity of the gene being studied. Indeed, a pooled analysis and another individual for each disease and for the samples will allow us to determine the number of base pairs of each gene. The number of polymorphic sites, informative parsimonious sites and the rate of transitions / transversions (R) will be calculated using the MEGA 6 software [4]. Two standard indices of nucleotide variability will be estimated using the DnaSP version 5.10.01 program [5]. These are haplotype diversity (h) and nucleotide diversity $(\pi)$. The haplotype diversity $(\mathrm{h})$ defines the probability that two randomly selected genes in a sample are identical [6] and takes into account the number of individuals as well as the haplotype frequency. The mean number of nucleotide differences (k) was released under DnaSP version 5.10.01 [5].

The transformation of the MMP9 nucleotide sequences into amino acid sequences is carried out using the MEGA6 software [4] by choosing the best reading frame (the one offering the least stop codons). The chi2 test has been used to see amino acids that show significant differences between healthy and cancerous tissues. The significance level of $5 \%$ has been retained.

We will study the differentiation and structuring of $M M P 9$ in this part. We will calculate the genetic distance of Nei [7] under MEGA 6 [4] using the Kimura 2-parameter model (K2P) with a bootstrap value of 10000 replications. Still under MEGA 6 [4] we will calculate Wright's Fst fixation index, (1969) using the software ARLEQUIN version 3.0 [8] thus measuring the heterozygote deficit between populations and reflects genetic differentiation. The P-value allows to accept the value of the Fst when it is significant $(\mathrm{P}<0.05)$ or to reject it when it is not significant $(\mathrm{P}>0.05)$ under neutrality. The existence of a deviation from the hypothesis of neutrality of the patients was studied by calculating the indices of D of Tajima [9], and $\mathrm{F} *$ of $\mathrm{Fu}$ and $\mathrm{Li}$ [10].

The homogeneity test or the natural selection test allow us to evaluate the neutrality (under selection or not) of mutations. They will be done with DnaSP version 5.10.01 [5] and MEGA 6 [4] with a bootstrap value of 10000 replications. We will evaluate the synonymous substitutions (dS) and non-synonymous substitutions (dN). The value of this neutrality will be accepted with a $\mathrm{P}$-value $<0.05$.

\section{Results}

\subsection{Analysis of the Polymorphism and of the MMP9 Gene Diversity}

The MMP9 gene reveals a high polymorphism for cataract and less for retinoblastoma and glaucoma. The transversion rates are significantly higher than those of transition except in the case of glaucoma where the transversion rates are significantly lower. We have a high haplotype diversity and low nucleotide diversity. The values are listed in table 1 and table 2 .

Table-1. Parameters of variability of $M M P 9$ sequences

\begin{tabular}{l|l|l|l}
\hline & Controls (Cs) & Glaucoma (Gc) & Cataract (Ct) \\
\hline Polymorphics sites & 0 & 2 & 23 \\
\hline Parsymonious sites & 0 & 1 & 17 \\
\hline Singletons sites & 0 & 1 & 6 \\
\hline Transition rate (Trs) en \% & 33.34 & 99.6 & 39.01 \\
\hline Transversion rate (Trv) en \% & 66.68 & 0.4 & 61 \\
\hline Mutation number (Eta) & 0 & 2 & 33 \\
\hline Haplotype number (h) & 1 & 3 & 17 \\
\hline Haplotype diversity (Hd) & 0 & $0.524+/-0.209$ & $0.713+/-0.08$ \\
\hline Nucleotide diversity (Pi) & 0 & $0.00740+/-0.00793$ & $0.05339+/-0.00796$ \\
\hline Nucleotide mean difference (K) & 0 & 0.762 & 5.499 \\
\hline
\end{tabular}

Table-2. Transition / Transversion

\begin{tabular}{l|l|l|c|l}
\hline & Groups & Controls & Glaucoma & Cataract \\
\hline Transition rate (Trs) en \% & 46.15 & 33.34 & 99.6 & 39.01 \\
\hline Transversion rate (Trv) en \% & 53.86 & 66.68 & 0.4 & 61 \\
\hline P-value & $<.0001$ & $<.0001$ & $<.0001$ & $<.0001$ \\
\hline
\end{tabular}

Molecular distances are relatively great for Glaucoma in the first position whereas for cataracts it is in the second position. Values are recorded in Table 3. 
Table-3. Molecular distance

\begin{tabular}{l|l|l|l|l}
\hline & Distance & $\mathbf{d ~ 1}^{\text {st }}$ Position & $\mathbf{d ~ 2}^{\text {nd }} \mathbf{P}$ & $\mathbf{d ~ 3}^{\text {rd }} \mathbf{P}$ \\
\hline Controls & 0 & 0 & 0 & 0 \\
\hline Glaucoma & $0,008+/-0,005$ & $0,014+/-0,015$ & $0,009+/-0,009$ & 0 \\
\hline Cataract & $0,212+/-0,035$ & $0,201+/-0,066$ & $0,238+/-0,065$ & $0,213+/-0,066$ \\
\hline
\end{tabular}

The nucleotide frequencies show for the group (cataract and glaucoma also) a slight superiority of $\% \mathrm{C}$ except in 2nd position or $\%$ A dominates. For the controls we have a domination of $\% \mathrm{G}$ except in 3rd position or $\% \mathrm{C}$ dominates. Table 4 illustrates this distribution. The relative values of nucleotide composition reveal a predominance of $\mathrm{C}+\mathrm{G}(51.6 \%)$ over $\mathrm{A}+\mathrm{T}(48.5 \%)$ in table 5 .

Table-4. Nucleotide frequency

\begin{tabular}{l|l|l|l|l}
\hline Frequencies (\%) & Groups & Controls & Glaucoma & Cataract \\
\hline T & 21.1 & 21.4 & 21.4 & 20.9 \\
\hline C & 31.7 & 27.2 & 29.1 & 33.3 \\
\hline A & 27.4 & 20.4 & 28.0 & 28.8 \\
\hline G & 19.9 & 31.1 & 21.5 & 17.0 \\
\hline T1 & 18 & 17 & 17 & 18 \\
\hline C1 & 37.4 & 22.9 & 40.0 & 40.2 \\
\hline A1 & 27.8 & 28.6 & 27.8 & 27.6 \\
\hline G1 & 17.2 & 31.4 & 15.1 & 14.4 \\
\hline T2 & 21 & 18 & 21 & 21 \\
\hline C2 & 23.4 & 26.5 & 20.6 & 23.3 \\
\hline A2 & 38.2 & 23.5 & 38.7 & 41.5 \\
\hline G2 & 17.8 & 32.4 & 20.2 & 14.0 \\
\hline T3 & 25 & 29 & 26 & 24 \\
\hline C3 & 34.1 & 32.4 & 26.5 & 36.1 \\
\hline A3 & 16.1 & 8.8 & 17.6 & 17.4 \\
\hline G3 & 24.6 & 29.4 & 29.4 & 22.6 \\
\hline
\end{tabular}

Table-5. Nucleotide frequency

\begin{tabular}{l|l|l|l|l|l|l|l|l}
\hline \multicolumn{1}{c}{ Frequencies (\%) } \\
\hline & $\mathrm{A}+\mathrm{T}$ & $\mathrm{C}+\mathrm{G}$ & $\mathrm{A}+\mathrm{T}$ & $\mathrm{C}+\mathrm{G}$ & $\mathrm{A}+\mathrm{T}$ & $\mathrm{C}+\mathrm{G}$ & $\mathrm{A}+\mathrm{T}$ & $\mathrm{C}+\mathrm{G}$ \\
\hline Groups & 48.5 & 51.6 & 45.8 & 54.6 & 59.2 & 41.2 & 41.1 & 58.7 \\
\hline Controls & 41.8 & 58.3 & 45.6 & 54.3 & 41.5 & 58.9 & 37.8 & 61.8 \\
\hline Glaucoma & 49.4 & 50.6 & 44.8 & 55.1 & 59.7 & 40.8 & 43.6 & 55.9 \\
\hline Cataract & 49.7 & 50.3 & 45.6 & 54.6 & 62.5 & 37.3 & 41.4 & 58.7 \\
\hline
\end{tabular}

The relative amino acid composition table 6 reveal several trends in patients:

- a significant decrease in frequency

- a significant increase

- a synthesis of new amino acids

- a suppression of the synthesis of others.

There is an equal frequency between controls and patients with retinoblastoma with the exception of alanine and valine.

Table-6. Amino acids frequency

\begin{tabular}{l|l|l|l|l|l}
\hline Non essentials Aminoacid (NE) & Group & Controls & Glaucoma & Cataract & P-value \\
\hline Ala & 8.614 & 15.151 & 8.928 & 7.155 & $<.0001$ \\
\hline Cys & 3.086 & 3.030 & 3.13 & 3.091 & $<.0001$ \\
\hline Asp & 5.987 & 0 & 9.38 & 6.713 & $<.0001$ \\
\hline Glu & 0.987 & 6.060 & 0 & 0 & $<.0001$ \\
\hline Gly & 0.740 & 3.030 & 0 & 0.353 & 0.132 \\
\hline His & 3.765 & 0 & 3.13 & 4.770 & 0.0012 \\
\hline Asn & 0.185 & 0 & 0 & 0.265 & 0.0010 \\
\hline Pro & 14.814 & 9.090 & 15.63 & 15.989 & $<.0001$ \\
\hline Gln & 0.123 & 0 & 0 & 0.176 & 0.0002 \\
\hline Arg & 6.913 & 6.060 & 9.38 & 6.625 & 0.2165 \\
\hline Ser & 11.543 & 12.121 & 15.63 & 10.600 & 0.0114 \\
\hline Tyr & 0.864 & 0 & 0 & 1.236 & 0.3089 \\
\hline Essentials Aminoacid & Group & Controls & Glaucoma & Cataract & P-value \\
\hline Lle & 5.432 & 3.030 & 6.3 & 5.830 & 0.0823 \\
\hline Leu & 11.049 & 18.181 & 9.38 & 9.717 & $<.0001$ \\
\hline Lys & 3.086 & 3.030 & 3.13 & 3.091 & $<.0001$ \\
\hline Met & 2.283 & 0 & 3.13 & 2.650 & 0.1208 \\
\hline Phe & 5.308 & 0 & 6.3 & 6.360 & $<.0001$ \\
\hline Thr & 11.851 & 6.060 & 3.571 & 14.840 & $<.0001$ \\
\hline Trp & 0.987 & 6.060 & 0 & 0 & $<.0001$ \\
\hline Val & 2.345 & 9.090 & 3.13 & 0.530 & $<.0001$ \\
\hline
\end{tabular}




\subsection{Differentiation and Genetic Structuring of the $M M P 9$ Gene}

The Genetic differentiation of $M M P 9$ gives intrapopulation distance (d) low for glaucoma but quite important for cataract. Values are reported in Table 7. The inter-population distances bring together cataracts and glaucoma (Table 8). We have for the rest a considerable genetic distance.

Table-7. Genetic distance (d) at the intra-population level

\begin{tabular}{l|l|l|l}
\hline & Controls & Glaucoma & Cataract \\
\hline Distance d+/-Sd & 0 & $0.008+/-0.005$ & $0.057+/-0.014$ \\
\hline
\end{tabular}

Table-8. Genetic distance (d) at the inter-population level

\begin{tabular}{l|l|l|c}
\hline & Controls & Glaucoma & Cataract \\
\hline Controls & - & & \\
\hline Glaucoma & $0.720+/-0.140$ & - & \\
\hline Cataract & $0.754+/-0.141$ & $0.073+/-0.022$ & - \\
\hline
\end{tabular}

The degree of differentiation is significantly elevated between controls and glaucoma, between controls and cataracts too. Differentiation is on the other hand significantly lower between cataract and glaucoma. The $p$ values of Tajima and Fs are not significant for both glaucoma and cataract (table 9).

Table-9. D of Tajima and Fs of Fu

\begin{tabular}{l|l|l|l|l}
\hline & D de Tajima & P-value D & Fs & P-value Fs \\
\hline Controls & 0 & 1 & N.A & N.A \\
\hline Glaucoma & -0.27492 & 0.364 & -0.43769 & 0.157 \\
\hline Cataract & -0.05277 & 0.533 & -3.74592 & 0.087 \\
\hline
\end{tabular}

\subsection{Homogeneity Test}

The average non-synonymous substitution number $(\mathrm{dN})$ is significantly greater than the average synonymous substitution number $(\mathrm{dS})$ for glaucoma.

Table-10. Demographic test

\begin{tabular}{l|l|l|l|l}
\hline & Groups & Controls & Glaucoma & Cataract \\
\hline Ds & $0.276+/-0.117$ & 0 & 0 & $0.012+/-0.007$ \\
\hline Dn & $0.245+/-0.052$ & 0 & $0.004+/-0.004$ & $0.066+/-0.018$ \\
\hline P-values & 0.035 & N/A & 0.0045 & 0.0609 \\
\hline
\end{tabular}

The mutation surveyor software Vs5.1.0 allowed us to highlight the mutations of the MMP9 gene in cataract and glaucoma. The following table presents these mutations.

Table-11. MMP9 gene mutations in cataract and glaucoma

\begin{tabular}{l|l|l}
\hline Patients & Mutation & \multicolumn{1}{c}{ Type } \\
\hline S3 & $1090 \mathrm{~T}>\mathrm{C}$ & $\mathrm{dbSNP}: 3918249$ \\
\hline $\mathrm{K} 7$ & $1090 \mathrm{~T}>\mathrm{TC} \quad$ 1097C $>$ CG & $\mathrm{dbSNP}: 3918249$ \\
\hline $\mathrm{K} 9$ & $1090 \mathrm{~T}>\mathrm{C}$ & $\mathrm{dbSNP}: 3918249$ \\
\hline $\mathrm{K} 18$ & $1090 \mathrm{~T}>\mathrm{C}$ & $\mathrm{dbSNP}: 3918249$ \\
\hline $\mathrm{K} 27$ & $1171 \mathrm{G}>\mathrm{GA}$ & $\mathrm{dbSNP}: 6104425$ \\
\hline $\mathrm{K} 28$ & $1090 \mathrm{~T}>\mathrm{TC}$ & $\mathrm{dbSNP}: 3918249$ \\
\hline $\mathrm{K} 29$ & $1090 \mathrm{~T}>\mathrm{C}$ & $\mathrm{dbSNP}: 3918249$ \\
\hline $\mathrm{K} 30$ & $1090 \mathrm{~T}>\mathrm{TC}$ & $\mathrm{dbSNP}: 3918249$ \\
\hline $\mathrm{K} 31$ & $1090 \mathrm{~T}>\mathrm{C}$ & $\mathrm{dbSNP}: 3918249$ \\
\hline $\mathrm{K} 32$ & $1171 \mathrm{G}>\mathrm{GA}$ & $\mathrm{dbSNP}: 6104425$ \\
\hline $\mathrm{K} 33$ & $1090 \mathrm{~T}>\mathrm{C}$ & $\mathrm{dbSNP}: 3918249$ \\
\hline $\mathrm{K} 15, \mathrm{~K} 27$ & $1185 \mathrm{G}>\mathrm{GA}$ & \\
\hline $\mathrm{S} 5$ & $136432 \mathrm{~A}>\mathrm{T}$ & \\
\hline $\mathrm{K} 11$ & $\mathrm{SNP} 136433 \mathrm{C}>\mathrm{A}$ & \\
\hline
\end{tabular}

\section{Discussion}

An analysis of nucleotide variability in blood was performed in Senegalese patients with cataract and glaucoma with the MMP9 marker. We used the population genetics approach applied to DNA samples taken from affected blood and healthy blood. Blood is considered as a population, exhibiting genetic diversity when the disease is present. The neutral polymorphism of controls highlights the functional polymorphism of the three diseases. Indeed, the $M M P 9$ gene has a higher polymorphism in cataract than with glaucoma, however high. The transversion rate is greater than the transition for cataract and inferior for glaucoma. We have high haplotypic diversity and low nucleotide diversity. This is the signal for rapid population growth from a small, efficient, age-old population for a time sufficient for mutational h restoration, but too short for the accumulation of large sequence differences.

The Molecular distance is non-existent in healthy patients (controls); it is variable in position $\left(1^{\text {st }}, 2^{\text {nd }}\right.$ or $3^{\text {rd }}$ codons) according to the disease. $\% \mathrm{C}$ noted in cataract and glaucoma except in 2 nd position or $\% \mathrm{~A}$ is superior. For 
controls, $\% \mathrm{G}$ is high except in $3^{\text {rd }}$ position where $\mathrm{C} \%$ is higher. The percentage of $\mathrm{C}-\mathrm{G}$ is greater than $\%$ A-T except for cataract in position 2 .

Mbaye, et al. [11], noted this increase in valine in the case of breast cancer. However, previous studies Kubota, et al. [12] found an increase in alanine in breast cancer patients. In our study we have the mutation (A2V) representing a substitution of Alanine by Valine.

The synthesis decrease of an amino acid resulting from metabolism would be synonymous of a reduction in the level of synthesis catalysts (catabolism) or an increase in the degradation rate of this amino acid in another amino acid. However, the increase of amino acid resulting from anabolism would be due to a diet rich in this sense or to a new acquisition of an amine acid which would go from essential to non-essential. Recent researches showed that many non-essential amino acids play a critical role in cancer metabolism [13]. In our study, as in the case of Mbaye, et al. [11], aliphatic amino acids (Alanine and Valine) appear to play a major role in cataract proliferation.

The frequency of certain amino acids remains constant in our study namely Glutamic acid (NE), Arginine (NE), Threonine (E) and Tryptophan (E). Mbaye, et al. [11], noted a decrease in tryptophan. Trp plays an important role in the proliferation of $\mathrm{T}$ lymphocytes. $\mathrm{T}$ lymphocytes are the main players in immune rejection reactions that can lead to the elimination of cancer cells and on which the various immunotherapy approaches currently tested are based. In fact, the methods used aim to stimulate the immune system so that it recognizes and destroys tumor cells [11].

The role of Trp induces that of the MMP9; indeed, numerous in vitro studies have shown the involvement of this enzyme in the "three-dimensional" migration of various cell types such as macrophages, B and T-cell, eosinophils, neutrophils and tumor cells. The role of MMP-9 in metastatic scattering has recently been confirmed by the reduction in the number of experimental metastases observed in $M M P 9$ missing in mice [14].

The constancy of Trp in our study could be a cause of inhibition of the activity of $M M P 9$ and also a resistance front to cataractogenesis. In addition, bone morphogenesis protein 4 (BMP-4) and epidermal growth factor (EGF) inhibit the expression of metalloproteinase-9 (MMP-9) in cancer cells [15]. Previously, another study demonstrated that BMP-4 inhibits the expression of $M M P 9$ in retinal cells [16]. These studies highlight the resistance of growth factors to tumor progression, they are in line with our study because in juvenile cataract patients aged under 25 years are in the development phase. This will justify the weak polymorphism of the MMP9 gene with juvenile cataract.

A study revealed that the expression of EGR-1 gene infered in stromals cells which are in direct control with lymphomas thanks to the $E G F$.

In fact, in vivo and in vitro experiences showed that this $E G R-1$ expression induced by $E G F$ inhibits the $M M P 9$ and repress the tumoral progress of thymics lymphomas [17]. Further, more in-depth studies will confirm this.

In patients with cataract there is a significant increase of Cysteine and Proline (NE), Lysine and Threonine (E); a significant decrease of Alanine and Serine (NE), Leucine and Valine (E). A synthesis of new amino acids is noted (Aspartate, Histidine, Asparagine, Glutamine, Tyrosine (NE) and Phenylalanine (E)) as well as the deletion of others (Glutamate (NE) and Tryptophan (E)). The (W6T) and (A2T) mutations explain the increase of Threonine.

A canadian study revealed that the $M M P 9$ contributes in the formation of the previous cataracte subcapsular.indeed the TTF induced in the presence of the MMP9 would be the median of the epithelialmesenchymal-transition (EMT) and this latter involves the transformation of the epithelial cells of the lens. They become plates below the capsule [18]. So MMP9 play a not insignificant role in the lens opacification.

Growth factors as for them seem to have an inhibitive effect on the $M M P 9$ at the eye level. The senile cataract is the most met in the Senegalese population. Thus a strong activity of the MMP9 could be noted at the adults (Age > 40) related with the absence of growth factor. Further studies can bring clarification.

The CRYAA gene can be substituted for arginine by cysteine (R116C), responsible for a high molecular weight protein aggregation resulting from an abnormal oligomerization of $\alpha$ - and $\beta$-crystallins.

This mutation leads to a zonular cataract associated with a microcornea and a microphthalmia. This is consistent with the increase in Cysteine noted in our study.

Another study [19] highlights two non-synonymous mutations that go in the same direction with the variations observed in our study. Indeed mutations with gamma-crystalline (p.R48H) and GJA8 (p.L281C) disrupt the normal structure of the lens and thus causes its opacification.

These genes appear to produce the same effects on the lens as MMP9. Indeed, a similar mutation (R9H) is noted in our study.

In glaucoma patients, the same amino acids are suppressed as in cataracts; the synthesis of the same amino acids [Aspartate, Histidine (NE) and Phenylalanine (NE)]. Glaucoma is also associated with an increase of amino acids [Cysteine, Serine, Proline (NE) and Lysine (E)] on the one hand and the decrease of other amino acids [Alanine (NE) and Leucine, Threonine Valine (E)] on the other hand. The Glutamic acid is a precursor of the proline biosynthesis, in glaucomatous the overexpression of proline may be related to the removal of glutamic acid.

Awadalla, et al. [20], describe in Caucasians an amino acid change (Arginine to Glutamine) responsible for protein expression of MMP9 favorable to glaucoma with SNPs rs17576 and rs3918249. In addition, Thakur, et al. [21] highlight the relationship between the change in $M M P 9$ activity in the retina, the optic nerve, the aqueous humor, the trabeculum and glaucoma in the human eye. In the Chinese, the most likely locus for angle-closure glaucoma is rs3918254. However, in our study, the majority SNP for Senegalese is rs3918249 so it goes in the same direction as Awadalla, et al. [20] in Caucasians.

The intrapopulation genetic distance is low for retinoblastoma and glaucoma (two mutations: $1090 \mathrm{~T}>\mathrm{C}$ and 136432A > T, however, it is slightly elevated in cataract (6 mutations: 136433C>A, $1185 \mathrm{G}>\mathrm{GA}, 1171 \mathrm{G}>\mathrm{GA}$, 1097C> CG and $1090 \mathrm{~T}>\mathrm{C})$. The inter-population distance approximates on the one hand the glaucomatous to cataracts $(7 \%)$ and on the other hand the retinoblastoma to controls $(0.5 \%)$, it distances the others however $(>70 \%)$. 
The locus SNPs3918249 is involved in both cataract and glaucoma, the same mutations are present in both diseases $((\mathrm{S} 3 \mathrm{M})$ and $(\mathrm{R} 9 \mathrm{H}))$ this resemblance was rather raised in the frequency of amino acids Salil, et al. [22] have already highlighted the implication of a deletion in the TDRD7 gene in both cataract and glaucoma, which is in line with our study.

For the entire population studied, we have $\mathrm{dS}>\mathrm{dN}$ (normal evolution of the gene MMP9). However, each disease is under positive selection $(\mathrm{dS}<\mathrm{dN})$ which leads to an increase in the frequency of variations in the human race $\mathrm{dN}>\mathrm{dS}$ for immunoglobulins, the MHC proteins and the ribonucleases [23].

\section{Conclusion}

The polymorphism of the MMP9 gene is associated with the two eye diseases. Indeed, Micheal, et al. [24] have highlighted the notorious impact of $M M P 9$ in closed-angle and open-angle glaucoma in the Pakistani population. Specific mutations have been noted in the Senegalese population (R9H, S3M and W6T). Cong, et al. [25], has demonstrated the association between SNPs3918249 and glaucoma (closed angle) in the Pakistani population. In Caucasians, this same mutation is found [20]. The same mutation is found in the African population and more precisely in the Senegalese in our study. The Senegalese population also presents SNPs 6104425, SNP 136432 and SNP136433.

It should be noted that SNP3918249 is present in both glaucoma and cataracts. Further studies may shed light on this polymorphism of the $M M P 9$ gene that brings two diseases with different pathological expressions closer.

In short, the polymorphism of the MMP9 gene in glaucoma, cataract and retinoblastoma in the Senegalese population present a coherent association. As cataract could be juvenile, it finds a barrier to the invasive potential of opacification in BMP4 and EGF [15], hence the low polymorphism of MMP9 found. MMP9 could be a great option in the fight against juvenile cataract.

At last Cataract and Glaucoma have a certain genetic heterogeneity between them. Further studies can deepen this convergence between these two diseases.

\section{Acknowledgment}

The authors would like to thank all donors enrolled in the present study.

\section{References}

[1] Nagase, H. and WoEssner, J. F., 1999. "Matrix metalloproteinases." Journal of Biological Chemistry, vol. 274, pp. 21491-4.

[2] Reponen, P., Leivo, I., Sahlberg, C., Apte, S. S., Olsen, B. R., Thesleff, I., and Tryggvason, K., 1995. "92kd type iv collagenase and timp-3, but not 72-kda type iv collagenase or timp-1 or timp-2, are highly expressed during mouse embryo implantation." Developmental Dynamics, vol. 202, pp. 388-96.

[3] Reponen, P., Sahlberg, C., Munaut, C., Thesleff, I., and Tryggvason, K., 1994. "High expression of the $92-$ $\mathrm{kD}$ type IV collagenase (gelatinase B) in the osteoclast lineage during mouse development." Journal of Cell Biology, vol. 124, pp. 1091-1102.

[4] Tamura, K., Stecher, G., Peterson, D., Filipski, A., and Kumar, S., 2013. "Mega6: Molecular evolutionary genetics version 6.0." Molecular Biology and Evolution, vol. 30, pp. 2725-29.

[5] Rozas, J., Librado, P., Sánchez-Del Barrio, J. C., Messeguer, X., and Rozas, R., 2012. "Dnasp version 5 help contents [help file]." Available: http://www.ub.edu/dnasp/

[6] Nei, M., 1987. Molecular evolutionary genetics. New York: Columbia University Press.

[7] Nei, M., 1978. "Estimation of average heterozygosity and genetic distance from a small number of individuals." Genetics, vol. 89, pp. 583-90.

[8] Excoffier, L., 2006. Computational and molecular population genetics lab cmpg. Zoological Institute, University of Berne.

[9] Tajima, F., 1989. "Statistical method for testing the neutral mutation hypothesis by DNA polymorphism." Genetics, vol. 123, pp. 585-95.

[10] Fu, Y. X. and Li, W. H., 1993. "Statistical tests of neutrality of mutations." Genetics, vol. 133, pp. 693-709.

[11] Mbaye, F., Dem, A., Fall, M., Diop, G., Mbengue, B., Diallo, R. N., Niang, M. S., Kane, M., Ka, S., et al., 2014. "Genetic diversity of breast cancer in senegalese women: New insight from somatic mutations." Journal of Health Science, vol. 4, pp. 25-33.

[12] Kubota, A., Meguid, M. M., and Hitch, D. C., 1992. "Amino acid profiles correlate diagnostically with organ site in three kinds of malignant tumors." Cancer, vol. 69, pp. 2343-48.

[13] Phang, J. M., Liu, W., and Hancock, C., 2013. "Bridging epigenetics and metabolism: role of nonessential amino acids." Epigenetics, vol. 8, pp. 231-36.

[14] Itoh, T., Tanioka, M., and Matsuda, H., 1999. "Experimental metastasis is suppressed in MMP-9-deficient mice." Clinical and Experimental Metastasis, vol. 17, pp. 177-81.

[15] Laulan, N. B., 2016. Modulation de l'expression des gènes pro-métastatiques MMP-9 et galectine-7 dans les cellules cancéreuses. Université du Québec, Institut national de la recherche scientifique, Doctorat en virologie et immunologie, p. 150.

[16] Xu, J., Zhu, D., Sonoda, S., He, S., Spee, C., Ryan, S. J., and Hinton, D. R., 2012. "Over-expression of BMP4 inhibits experimental choroidal neovascularization by modulating VEGF and MMP-9." Angiogenesis, vol. 15, pp. 213-27. 
[17] Bouchard, F., Bélanger, S. D., Birion-Pain, K., and St-Pierre, Y., 2010. "EGR-1 activation by EGF inhibits MMP-9 expression and lymphoma growth." Blood, vol. 116, pp. 759-66.

[18] Korol, A., Pino, G., Dwivedi, D., Robertson, J. V., Deschamps, P. A., and West-Mays, J. A., 2014. "Matrix metalloproteinase-9-null mice are resistant to TGF- $\beta$-induced anterior subcapsular cataract formation." American Journal of Pathology, vol. 184, pp. 2001-12.

[19] Kumar, R., Parsad, D., Kanwar, A. J., and Kaul, D., 2011. "Altered levels of Ets-1 transcription factor and matrix metalloproteinases in melanocytes from patients with vitiligo." British Journal of Dermatology, vol. 165, pp. 285-91.

[20] Awadalla, M. S., Burdon, K. P., Kuot, A., Hewitt, A. W., and Craig, J. E., 2011. "Matrixmetalloproteinase9 genetic variation and primary angle closure glaucoma in a Caucasian population." Molecular Vision, vol. 17, pp. 1420-1424.

[21] Thakur, N., Kupani, M., Pandey, R. K., Mannan, R., Pruthi, A., and Mehrotra, S., 2018. "Genetic association of $-1562 \mathrm{C}>\mathrm{T}$ polymorphism in the MMP9 gene with primary glaucoma in a north Indian population." PLoS ONE, vol. 13, p. e0192636.

[22] Salil, G., Nevin, K. G., and Rajamohan, T., 2011. "Arginine rich coconut kernel protein modulates diabetes in alloxan treated rats." Chimico-Biological Interactions, vol. 189, pp. 107-11.

[23] Yang, Z., Nielsen, R., Goldman, N., and Pedersen, A. M., 2000. "Codon-substitution models for heterogeneous selection pressure at amino acid sites." Genetics, vol. 155, pp. 431-49.

[24] Micheal, S., Yousaf, S., Khan, M. I., Akhtar, F., Islam, F., Khan, W. A., den Hollander, A. I., Qamar, R., and Ahmed, A., 2013. "Polymorphisms in matrix metalloproteinases MMP1 and MMP9 are associated with primary open-angle and angle closure glaucoma in a Pakistani population." Molecular Vision, vol. 19, pp. 441-7.

[25] Cong, Y., Guo, X., Liu, X., Cao, D., Jia, X., Xiao, X., Li, S., Fang, S., and Zhang, Q., 2009. "Association of the single nucleotide polymorphisms in the extracellular matrix metalloprotease-9 gene with PACG in southern China." Molecular Vision, vol. 15, pp. 1412-7. 